\title{
Extramammary Paget's disease with the appearance of a nodule: a case report
}

Xia Wang, Wenlin Yang ${ }^{*}$, Jian Yang

\begin{abstract}
Background: Extramammary Paget's disease (EMPD) remains a rare condition with only a limited number of cases reported in the literature. EMPD is mainly composed of intraepidermal Paget cells, and possesses variable clinical behaviors and histological appearances, leading to difficulty in the diagnosis of this disease.

Case presentation: We here report a case of primary EMPD with the appearance of a nodule on the background of erythema. Histological assessment showed Paget cell infiltration throughout the epidermis with dermal spread. Using immunohistochemistry, the expressions of CK7, CK19, CK20, GCDFP-15, CEA, S-100 protein and bcl-2 were examined to elucidate the cellular differentiation of the carcinoma.
\end{abstract}

Conclusion: According to the histological assessment, this case was diagnosed as primary EMPD with carcinoma cells invading into the dermis, but without lymph node infiltration.

\section{Background}

EMPD of the vulva is a rare intraepithelial adenocarcinoma which accounts for less than $1 \%$ of carcinomas in vulva, while the majority of the patients are postmenopausal Caucasian females[1-3]. The cancer cells in the neoplasm usually stay "in situ" and only rarely invade into the dermis to be metastatic via the lymphatic system $[4,5]$. One has to differentiate neoplasms with Paget phenomenon from carcinomas metastasized from adjacent organs such as the urinary system and rectum [6]. Here, we present an unusual case of EMPD with carcinoma cells invading into the dermis without lymph node infiltration in a Chinese woman.

\section{Case presentation}

A 66-year-old Chinese woman presented with a slowly growing vulvar mass with pruritus. Two years prior to presentation, she noted a painless red firm nodule on her vulva. Subsequently, erythema around the nodule appeared. The nodule and erythema had gradually enlarged during the 2-year period. Physical examination revealed a $2 \mathrm{~cm} \times 2 \mathrm{~cm} \times 2 \mathrm{~cm}$ slightly erosive, nodular red firm mass on the centre of $5 \mathrm{~cm} \times 7 \mathrm{~cm}$ area of irregular eczematoid erythema covering her mons pubis

\footnotetext{
* Correspondence: wenliny@21cn.com

Department of Dermatology, the Second Affiliated Hospital of Guangzhou Medical University, Guangzhou 510260, P. R. China
}

(Fig.1). There was no swelling of lymph nodes in bilateral groins. Biopsy of inguinal lymph node was negative. The complete mass and part of the erythema were excised for histological evaluation. The patient's medical history was unremarkable. Metastasis from other cancers was excluded by routine blood tests, cystoscopy, oesophagogastroduodenoscopy, colonoscopy, abdominopelvic computed tomography $(\mathrm{CT})$, chest radiography and positron emission tomography-CT. All these examinations were normal. At 3-month follow-up, the patient had local mass recurrence and non-palpable inguinal lymph nodes.

Hematoxylin and Eosin staining of the biopsy from the lesion showed large round cells with ample pale-staining cytoplasm, pleomorphic nuclei, and occasional prominent nucleoli infiltrating throughout the epidermis, indicative of Paget cells (Fig. 2A). This carcinoma spread dermal, and a poorly differentiated adenocarcinoma was present in the dermis.(Fig. 2B). Immunohistochemical stainings for gross cystic disease fluid protein-15 (GCDFP-15) (Fig. 3A), cytokeratin7(CK7) (Fig. 3B), cytokeratin 19 (CK19) and carcinoembryonic antigen (CEA) were strongly positive. Epithelial membrane antigen (EMA) was positive, while S-100 protein, bcl-2 and CK20 (Fig. 3C) were all negative. Neoplastic cells were positive for periodic acid-Schiff (PAS). Moreover, the Paget cells and low-differentiated adenocarcinoma 


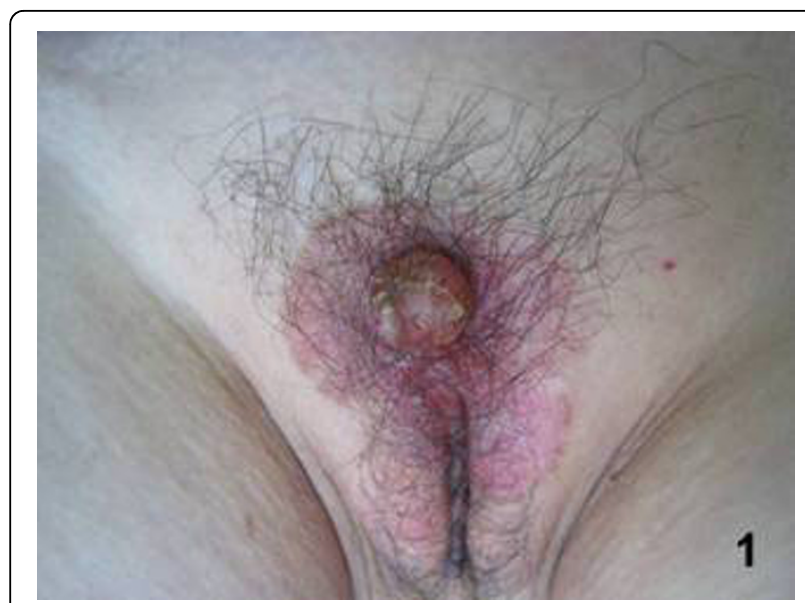

Figure 1 The clinical features of the patient with EMPD. Red firm mass on the centre of irregular warm eczematoid erythema covering the woman mons pubis.

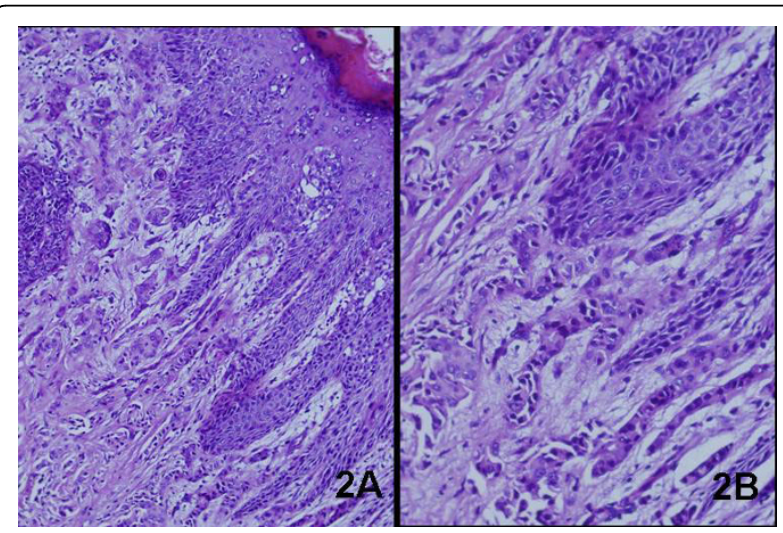

Figure 2 Histological assessment of EMPD. (A) H\&E staining indicated Paget cells into the epidermis (original magnification $\times$ 100), and (B) The dermis contained a poorly differentiated adenocarcinoma (original magnification $\times 200$ ). shared common immunohistological features. These immunohistochemical appearances supported the diagnosis of EMPD.

\section{Discussion}

EMPD is an intraepidermal adenocarcinoma, which usually develops in locations with apocrine glands. Nevertheless, when the carcinoma cells infiltrate into the dermis and form a nodule, this disease turns into an invasive carcinoma belonging to adnexal adenocarcinoma of the skin [7]. Typically involved anatomical sites are the vulvar, perianal, perineal, scrotal and penile regions. Clinically, the lesions present as well-defined, moist, erythematous plaques usually accompanied by pruritus. The study reported by Hatta et al [8] revealed that erythema was a clinical characteristic of all lesions; furthermore, nodules were present in $24 \%$, erosion in $49 \%$ and hypopigmentation in $25 \%$ cases. In addition, $39 \%$ of patients exhibited lymphopathy.

Primary adenocarcinomas of the vulva have been classified into sweat gland cancers, EMPD $[9,10]$, and"breast-like"adenocarcinomas of the vulva $[11,12]$. Histologically, sweat gland carcinomas of the vulva possess adenopapillary cords and tubules, occasionally comprising pagetoid cells. Although EMPD mainly consists of intraepidermal Paget cells, dermal invasion with cords and sheets has also been recorded [13]. The current opinion is that the malignancy spreads from superficial to deep [14], rather than from deep to superficial $[13,15]$. Primary breast carcinoma of the vulva exhibits histological characteristics similar to breast carcinoma. These three carcinomas may possess some consistent histopathological features. Van der Putte and van Gorp [16] put forward the term "adenocarcinoma of the mammary-like glands of the vulva", leading to a novel unifying notion for the three diseases mentioned above.

The level of invasion of the paget cells in EMPD can be classified into three grades: in situ in the epidermis, microinvasion to the papillary dermis, and deep invasion

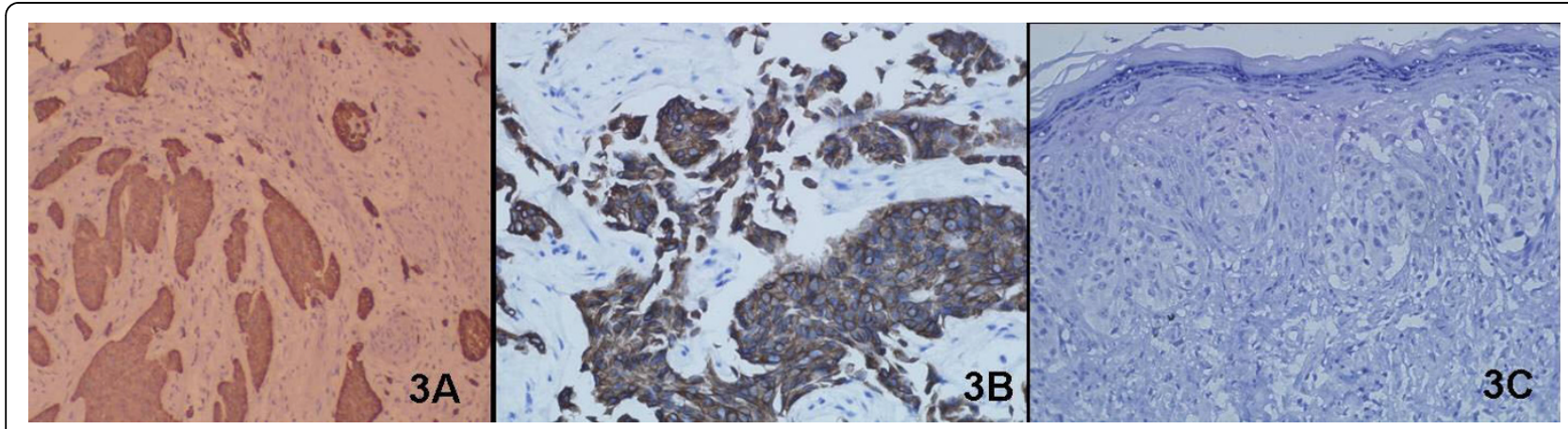

Figure 3 Immunohistochemical assessment of EMPD. (A) The carcinoma showed positivity for GCDFP-15(original magnification $\times 100)$.(B) The carcinoma showed positivity for CK7 (original magnification $\times 200$ ).(C) The carcinoma showed negative for CK20 (original magnification $\times 200$ ) 
into the reticular dermis or subcutaneous tissue [17]. According to the above classification method, this case can fall into the third grade. Hatta et al [8] reported that male patients outnumbered female patients (malefemale ratio, 2.6 to 1 ) in Japanese, in contrast to previous reports from western countries. The discrepancy between the studies can be explained by genetic variety of different regions. Further epidemiological studies for this disease are needed to clarify this point.

In this case, the lesion was present as a painless, slowly-growing, red firm mass on the centre of irregular eczematoid erythema covering the elderly woman's mons pubis. Histological assessment showed EMPD with carcinoma cells invading into the dermis, which are often characteristics of the metastatic lesion of breast. However, metastasis from breast carcinoma and internal malignancies was excluded by physical and auxiliary examinations. When carcinoma cells infiltrate into the dermis and advance to Paget's carcinoma, it is referred to as adenocarcinoma of the skin and has a poor prognosis. Up to now, no established guideline has been made for the diagnosis of EMPD, the nonspecific clinical findings of EMPD often lead to misdiagnosis and extended periods of topical and systemic medical mismanagement.

Because the disease is rare, there is little knowledge of the most effective treatment. Primary treatment is surgical and involves wide local excision with frozen section evaluation of margins. Invasion level and multiple lymph node metastases are important prognostic factors in EMPD. Unfortunately, there is a high rate of recurrence. Some suggest prophylactic regional lymph node dissection, especially for high grade carcinomas, whereas others recommend removal of only clinically involved nodes [9]. Reports on the results of radiotherapy are conflicting: these carcinomas are radioresistant, or radiotherapy increases local control [3]. Radiation therapy may be used as a supplement to aggressive surgery. We recommend that treatment for EMPD includes surgery in the form of wide local excision and adjuvant radiotherapy with caution and individualization.

\section{Conclusions}

Primary EMPD of the vulva is exceptional. Up to now, no gold diagnostic standard has been established for EMPD, leading to the difficulty in diagnosis of this disease. To improve its prognosis, further investigation needs to be performed for the early detection of primary EMPD and its metastasis.

\section{Consent}

Written informed consent was obtained from the patient for publication of this case report and any accompanying images. A copy of the written consent is available for review by the Editor-in-Chief of this journal.

\section{Abbreviations}

EMPD: Extramammary Paget's disease; CT: computed tomography; GCDFP15: gross cystic disease fluid protein-15; CK7: cytokeratin 7; CK19: cytokeratin 19; CK20: cytokeratin 20; CEA: carcinoembryonic antigen; EMA: epithelial membrane antigen; bcl-2: b cell lymphoma/lewkmia-2; PAS: periodic acidschiff

\section{Acknowledgements}

We are grateful to Dr. Zhuofang Hao, Dr. Shizhang Huang and Dr. Degui Liao in the Pathology Department of our hospital for their contribution to this project. This work was supported by Science and Technology Planning Project of Guangdong Province, P R. China (No. 2008B030301249).

\section{Authors' contributions}

XW drafted the manuscript and in the data collection. WLY participated in the design and coordination of the case-study and revised the manuscript for important intellectual content. JY carried out the histological examination and diagnosed, investigated, followed up and managed the patient. All authors have read and approved the final manuscript.

\section{Authors' information}

Xia Wang, Resident Doctor, Department of Dermatology, the Second Affiliated Hospital of Guangzhou Medical University, Guangzhou 510260, P. R. China. E-mail: wxpiglet@yahoo.com.cn.

WenLin Yang, Professor, Department of Dermatology, the Second Affiliated Hospital of Guangzhou Medical University, Guangzhou 510260, P. R. China. Email: wenliny@21cn.com.

Jian Yang, Professor, Department of Dermatology, the Second Affiliated Hospital of Guangzhou Medical University, Guangzhou 510260, P. R. China. Email: Yangj123@21cn.com.

\section{Competing interests}

The authors declare that they have no competing interests.

Received: 20 March 2010 Accepted: 4 August 2010

Published: 4 August 2010

\section{References}

1. Pitman GH, McCarthy JG, Perzin KH, Herter FP: Extramammary Paget's disease. Plast Reconstr Surg 1982, 69(2):238-44.

2. Zollo JD, Zeitouni NC: The Roswell Park Cancer Institute experience with extramammary Paget's disease. Br J Dermatol 2000, 142(1):59-65.

3. Besa P, Rich TA, Delclos L, Edwards CL, Ota DM, Wharton JT: Extramammary Paget's disease of the perineal skin: role of radiotherapy. Int J Radiat Oncol Biol Phys 1992, 24(1):73-8.

4. Park S, Grossfeld GD, McAninch JW, Santucci R: Extramammary Paget's disease of the penis and scrotum: excision, reconstruction and evaluation of occult malignancy. J Urol 2001, 166(6):2112-6.

5. de Blois GG, Patterson JW, Hunter SB: Extramammary Paget's disease: Arising in knee region in association with sweat gland carcinoma. Arch Pathol Lab Med 1984, 108(9):713-6.

6. Alsaad KO, Obaidat N, Dube V, Chapman W, Ghazarian D: Vulvar apocrine adenocarcinoma: a case with nodal metastasis and intranodal mucinous differentiation. Pathol Res Pract 2009, 205(2):131-5.

7. McCarter MD, Quan SH, Busam K, Paty PP, Wong D, Guillem JG: Long-term outcome of perianal Paget's disease. Dis Colon Rectum 2003, 46(5):612-6.

8. Hatta N, Yamada M, Hirano T, Fujimoto A, Morita R: Extramammary Paget's disease: treatment, prognostic factors and outcome in 76 patients. $\mathrm{Br} J$ Dermatol 2008, 158(2):313-8.

9. Lee SC, Roth LM, Ehrlich C, Hall JA: Extramammary Paget's disease of the vulva. A clinicopathologic study of 13 cases. 1977, 39(6):2540-9.

10. Roth LM, Lee SC, Ehrlich CE: Paget's disease of the vulva. A histogenetic study of five cases including ultrastructural observations and review of the literature. Am J Surg Pathol 1977, 1(3):193-206

11. Guerry RL, Pratt-Thomas HR: Carcinoma of supernumerary breast of vulva with bilateral mammary. 1976, 38:2570-2574. 
12. Simon KE, Dutcher JP, Runowicz CD, Wiernik PH: Adenocarcinoma arising in vulvar breast tissue. 1988, 62(10):2234-8.

13. Jones RE, Austin C, Ackerman AB: Extramammary Paget's disease. A critical reexamination. Am J Dermatopathol 1979, 1(2):101-32.

14. Merot Y, Mazoujian G, Pinkus G, Momtaz-T K, Murphy GF: Extramammary Paget's disease of the perianal and perineal regions. Evidence of apocrine derivation. Arch Dermatol 1985, 121(6):750-2.

15. Lloyd J, Flanagan AM: Mammary and extramammary Paget's disease. J Clin Pathol 2000, 53(10):742-9.

16. Van der Putte SCJ, van Gorp LHM: Adenocarcinoma of the mammary-like glands of the vulva: a concept unifying sweat gland carcinoma of the vulva, carcinoma of supernumerary mammary glands and extramammary Paget's disease. J Cutan Pathol 1994, 21(2):157-63.

17. Hatta N, Morita R, Yamada M, Echigo T, Hirano T, Takehara K, Ichiyanagi K, Yokoyama K: Sentinel lymph node biopsy in patients with extramammary Paget's disease. Dermatol Surg 2004, 30(10):1329-34.

Pre-publication history

The pre-publication history for this paper can be accessed here: http://www.biomedcentral.com/1471-2407/10/405/prepub

doi:10.1186/1471-2407-10-405

Cite this article as: Wang et al.: Extramammary Paget's disease with the appearance of a nodule: a case report. BMC Cancer 2010 10:405.

\section{Submit your next manuscript to BioMed Central} and take full advantage of:

- Convenient online submission

- Thorough peer review

- No space constraints or color figure charges

- Immediate publication on acceptance

- Inclusion in PubMed, CAS, Scopus and Google Scholar

- Research which is freely available for redistribution

Submit your manuscript at www.biomedcentral.com/submit 